\title{
FRATURA TIPO EXPLOSÃO DA COLUNA TORÁCICA E LOMBAR: CORRELAÇÃO ENTRE O SEGMENTO BIOMECÂNICO SAGITAL ACOMETIDO E AS ALTERAÇÕES ESTRUTURAIS DA VÉRTEBRA FRATURADA
}

\author{
BURST FRACTURE OF THE THORACIC AND LUMBAR SPINE: CORRELATION BETWEEN THE \\ BIOMECHANICAL SAGITTAL SEGMENT AFFECTED AND STRUCTURAL \\ CHANGES OF THE FRACTURED VERTEBRA
}

\author{
FRACTURA POR ESTALLIDO DE LA COLUMNA TORÁCICA Y LUMBAR: CORRELACIÓN \\ ENTRE EL SEGMENTO BIOMECÁNICO SAGITAL AFECTADO Y LOS CAMBIOS \\ ESTRUCTURALES DE LA VÉRTEBRA FRACTURADA
}

Matheus luís da Silva ${ }^{1}$, Rodrigo Arnold Tisot $^{1}$, Juliano Silveira Luiz Vieira ${ }^{1}$, Renato Tadeu dos Santos ${ }^{1}$, Orley Fauth Tisot ${ }^{1}$

\begin{abstract}
RESUMO
Objetivo: Analisar as fraturas tipo explosão da coluna torácica e lombar e fazer a correlação entre o segmento biomecânico sagital acometido e as alterações estruturais da vértebra fraturada. Métodos: Estudo retrospectivo de 72 pacientes com fraturas tipo explosão da coluna torácica e lombar. O estreitamento do canal vertebral, o colapso vertebral e a cifose local foram avaliados em três segmentos distintos: torácico, transição toracolombar e lombar. Resultados: Houve diferença estatística significativa $(p<0,05)$ dos valores do estreitamento do canal vertebral e cifose local nos diferentes segmentos da coluna vertebral avaliados. Conclusão: As fraturas tipo explosão da coluna torácica e lombar, embora apresentem características semelhantes, independentemente do local de ocorrência, sofrem influência direta do segmento biomecânico sagital no que diz respeito às alterações estruturais que ocorrem na vértebra fraturada.
\end{abstract}

Descritores: Fraturas da coluna vertebral; Traumatismos da coluna vertebral; Cifose; Canal vertebral; Biomecânica.

\begin{abstract}
Objective: To analyze the thoracic and lumbar burst fractures and make a correlation of the biomechanical sagittal segment affected with the structural changes of the fractured vertebra. Methods: A retrospective study of 72 patients with thoracic and lumbar burst fractures. The narrowing of the spinal canal, vertebral collapse and local kyphosis were evaluated in three distinct segments: thoracic, thoracolumbar transition, and lumbar. Results: There was a statistically significant difference $(p<0.05)$ in the spinal canal narrowing and kyphosis in the different segments of the spine evaluated. Conclusion: Burst fractures of the thoracic and lumbar spine, although with similar characteristics regardless of where they occur, are influenced directly by the biomechanical sagittal segment with respect to structural changes that occur in the fractured vertebra.
\end{abstract}

Keywords: Spinal fractures; Spinal injuries; Kyphosis; Spinal canal; Biomechanics.

RESUMEN

Objetivo: Analizar las fracturas por estallido de la columna torácica y lumbar y hacer una correlación del segmento biomecánico sagital afectado con los cambios estructurales de la vértebra fracturada. Métodos: Estudio retrospectivo de 72 pacientes con fracturas por estallido en la columna torácica y lumbar. El estrechamiento del canal espinal, el colapso vertebral y la cifosis fueron evaluados en tres segmentos distintos: torácico, transición toracolumbar y lumbar. Resultados: Se observó una diferencia estadísticamente significativa ( $p$ <,05) en el estrechamiento del canal espinal y la cifosis en diferentes segmentos de la columna vertebral evaluados. Conclusión: Las fracturas torácica y lumbar por estallido, aunque tienen características similares independientemente del lugar donde se producen, son directamente influenciadas por el segmento biomecánico sagital con respecto a los cambios estructurales que ocurren en la vértebra fracturada.

Descriptores: Fracturas de la columna vertebral; Traumatismos vertebrales; Cifosis; Conducto vertebral; Biomecánica.

INTRODUÇÃO

A fratura da coluna vertebral torácica e lombar tipo explosão é o resultado da falência do corpo vertebral em sua coluna anterior e média quando submetido à pressão no eixo axial. ${ }^{1}$ Ela apresenta frequente associação com dano neurológico e ocorre, geralmente, em indivíduos jovens envolvidos com trauma de grande impacto.

As alterações radiográficas típicas dessa fratura são o afastamento pedicular do arco vertebral na incidência anteroposterior, a diminuição da altura vertebral na incidência em perfil. Além disso, há retropulsão de fragmentos do corpo vertebral para dentro do canal vertebral. ${ }^{1,2} \mathrm{O}$ fragmento projetado para dentro do canal vertebral geralmente ocorre na metade superior da vértebra, onde o posi- cionamento do pedículo do arco vertebral, na radiografia simples, dificulta sua visibilização. ${ }^{1,3}$

O crescente número de acidentes industriais e automobilísticos tem influência direta no aumento do número de lesões complexas e graves da coluna vertebral. Nos pacientes politraumatizados a incidência é ainda maior, sendo a associação com lesões neurológicas grande. Nas últimas décadas, com o advento de novas tecnologias para o diagnóstico preciso e tratamento das referidas lesões, o estudo das características individuais de cada tipo de fratura tem influenciado na estratégia terapêutica. ${ }^{4-8}$

Mesmo com o advento crescente de tecnologias que permitem um diagnóstico e condutas terapêuticas mais coerentes, o

1. Grupo de Coluna do Hospital Ortopédico de Passo Fundo, Passo Fundo, RS, Brasil.

Trabalho realizado no Serviço de Residência Médica de Ortopedia eTraumatologia do Hospital Ortopédico de Passo Fundo, Passo Fundo, RS, Brasil. Correspondência: Rua Passo Fundo, 456 - Centro, 99700-000. Erechim, RS, Brasil.mlsilva7@gmail.com 
tratamento das fraturas da coluna torácica e lombar tipo explosão é ainda um tema controverso, trazendo diferentes opiniões com relação às indicações do tratamento conservador ou cirúrgico..$^{9-11}$

As características biomecânicas sagitais da coluna vertebral, embora muitas vezes desprezadas durante a avaliação diagnóstica e definição terapêutica, possuem valor significativo na determinação da gravidade da lesão. Embora o tipo de fratura seja importante para determinar a conduta terapêutica, é imprescindível que se distinga o segmento da coluna vertebral em que a fratura ocorreu. O segmento torácico encontra-se em cifose e, a transição toracolombar $\left(T_{12}-L_{1}\right)$, também em cifose ou em transição para lordose. Já as vértebras lombares $\left(L_{2}, L_{3}, L_{4}\right.$ e $\left.L_{5}\right)$, encontram-se em área de lordose. ${ }^{12,13}$ Desta forma, o trauma axial, que causa a fratura do tipo explosão, pode provocar diferentes alterações estruturais na vértebra fraturada, conforme o segmento vertebral acometido. ${ }^{14}$

As características anatômicas dos diferentes segmentos da coluna vertebral também influenciam na incidência e alterações estruturais que poderão estar presentes na vértebra fraturada. No segmento torácico as vértebras têm o formato de ligeira cunha configurando com os discos intervertebrais sua cifose fisiológica. As costelas estão conectadas por ligamentos, formando a caixa torácica que acaba fornecendo rigidez ao segmento. A região de transição toracolombar, possui uma característica anatômica importante, que é a transição de uma suposta coluna vertebral mais rígida para uma coluna mais flexível. ${ }^{15}$ Os fatores anatômicos de importância no segmento lombar são a grande robustez das vértebras, os ligamentos mais espessos, o canal vertebral que possui maior área para acomodar o tecido nervoso e a estabilização adicional é propiciada pelos músculos paravertebrais lombares e o iliopsoas. ${ }^{13}$

O objetivo desse estudo é analisar as fraturas da coluna torácica e lombar do tipo explosão e efetuar uma correlação do segmento biomecânico sagital acometido com as alterações estruturais da vértebra fraturada.

\section{MATERIAIS E MÉTODOS}

Foi realizado um estudo retrospectivo, por meio da análise de prontuários dos Serviços de Arquivos Médicos do Hospital São Vicente de Paulo de Passo Fundo (SAME - HSVP) e do Hospital da Cidade de Passo Fundo (SAME - HCPF), de 86 pacientes com fraturas da coluna vertebral torácica e lombar tipo explosão, no período compreendido entre abril de 2002 e outubro de 2009.

Foram considerados critérios de exclusão, os pacientes que apresentavam fratura tipo explosão com mais de três semanas de evolução, fraturas em mais de uma vértebra e fraturas em osso patológico.

Dessa forma, 72 pacientes com fraturas da coluna torácica e lombar tipo explosão preencheram os requisitos necessários para a pesquisa.

Todas as fraturas estavam documentadas com radiografias da coluna vertebral nas incidências anteroposterior e perfil, além dos filmes de tomografia computadorizada (TC) com cortes axiais interessando tecido ósseo e partes moles.

Para avaliação das alterações estruturais da vértebra fraturada, conforme o segmento biomecânico sagital em que a lesão ocorreu, as fraturas foram agrupadas em três grupos: torácicas $\left(T_{1} a T_{11}\right)$, transição toracolombar $\left(T_{12}\right.$ e $\left.L_{1}\right)$, e lombares $\left(L_{2}\right.$ a $\left.L_{5}\right)$.

As seguintes alterações estruturais, nas fraturas da coluna torácica e lombar tipo explosão, foram mensuradas: grau de cifose, porcentagem de colapso da parede anterior do corpo vertebral e porcentagem de estreitamento do canal vertebral.

O grau de cifose foi mensurado nas radiografias em incidência perfil, através do método descrito por Cobb. ${ }^{16}$

Para determinar a porcentagem do colapso da parede anterior do corpo vertebral de cada uma das vértebras fraturadas, foi utilizado o método de Willen et al. ${ }^{17} \mathrm{~A}$ fórmula estima a altura que o corpo vertebral apresentava antes da ocorrência da fratura.
A técnica de aferição do estreitamento do canal vertebral pelo fragmento ósseo projetado para o seu interior, na TC axial com janela óssea, foi realizada com régua transparente graduada em milímetros e baseada no diâmetro sagital médio. O diâmetro do canal vertebral normal, antes do trauma, foi estimado pela média dos valores encontrados nos cortes axiais no correspondente anatômico das vértebras adjacentes ao local da fratura. ${ }^{17}$

Para a análise estatística, foi utilizado a estatística descritiva e o teste não-paramétrico de Mann-Whitney. O nível de significância de $0,05(\alpha=5 \%)$ e os níveis descritivos (p) inferiores a esse valor foram considerados significativos e representados por $p<0,05$. Todas as análises foram realizadas pelo software SPWA para Windows versão 18.

\section{RESULTADOS}

Dos 72 pacientes avaliados, 48 (66,7\%) eram do sexo masculino e $24(33,3 \%)$ do sexo feminino e a idade variou de 12 a 79 anos (média de 41,4 anos).

Os mecanismos de trauma encontrados foram: queda de altura em 49 (68\%) pacientes, acidente automobilístico em 11 (15,3\%), acidente de motocicleta em 7 (9,7\%), atropelamento em $1(1,4 \%)$, soterramento em $3(4,2 \%)$ e outros em 1 (1,4\%). (Figura 1)

Com relação ao nível da fratura, a maioria dos casos ocorreu na transição toracolombar, sendo a vértebra com mais incidência a $L_{1}$, com 28 casos $(38,9 \%)$. (Figura 2$)$

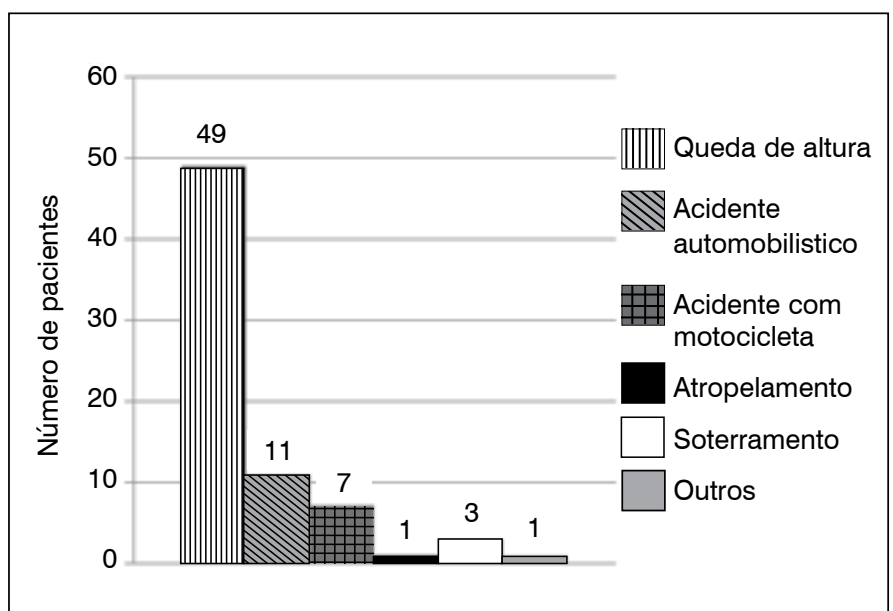

Figura 1. Distribuição dos pacientes quanto à etiologia do trauma que acarretou as fraturas da coluna torácica e lombar tipo explosão.

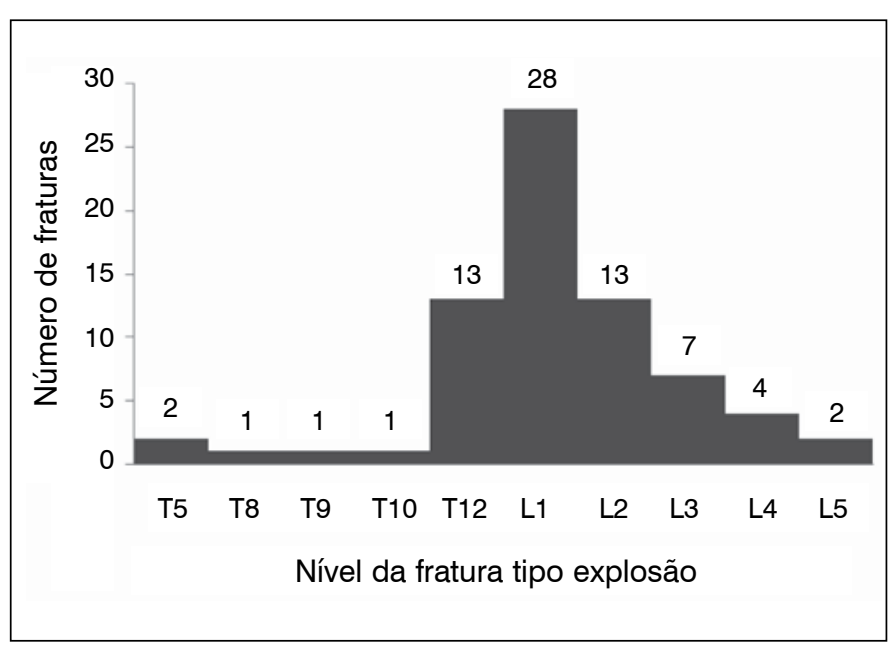

Figura 2. Distribuição das fraturas torácica e lombar tipo explosão. 
Quanto a classificação de Magerl et al. ${ }^{18}$, houve 49 (68\%) pacientes com fraturas A3.1, 8 (11,1\%) A3.2 e 15 (22,9\%) A3.3.

De acordo com o exame neurológico na admissão hospitalar, houve 2 (2,8\%) pacientes com Frankel B, 1 (1,4\%) paciente com Frankel C, 7 (9,7\%) pacientes com Frankel D e $62(86,1 \%)$ pacientes com Frankel E.

Com relação ao segmento biomecânico sagital, a maioria das fraturas tipo explosão ocorreu na transição toracolombar, com 41 pacientes $(56,9 \%)$. No segmento lombar, houve $26(36,1 \%)$ fraturas e, no segmento torácico, 5 (7\%) casos. (Figura 3)

Segundo o método de avaliação utilizado, a porcentagem média do estreitamento do canal vertebral foi de $32,8 \%$ e variou de $5 \%$ a 95\%. (Tabela 1)

$\mathrm{Na}$ avaliação da porcentagem média de colapso do corpo vertebral, foi obtida uma média de $34,3 \%$, variando de $0,4 \%$ a 82,4\%. (Tabela 2)

Já a média do grau de cifose, na região fraturada, foi de $9,8^{\circ}$ e variou de $-30^{\circ}$ até $40^{\circ}$. A média de cifose de cada um dos grupos de fraturas, analisados conforme o segmento biomecânico sagital, foi comparado e obteve diferença estatística significativa $(p<0,05)$. (Tabela 3)

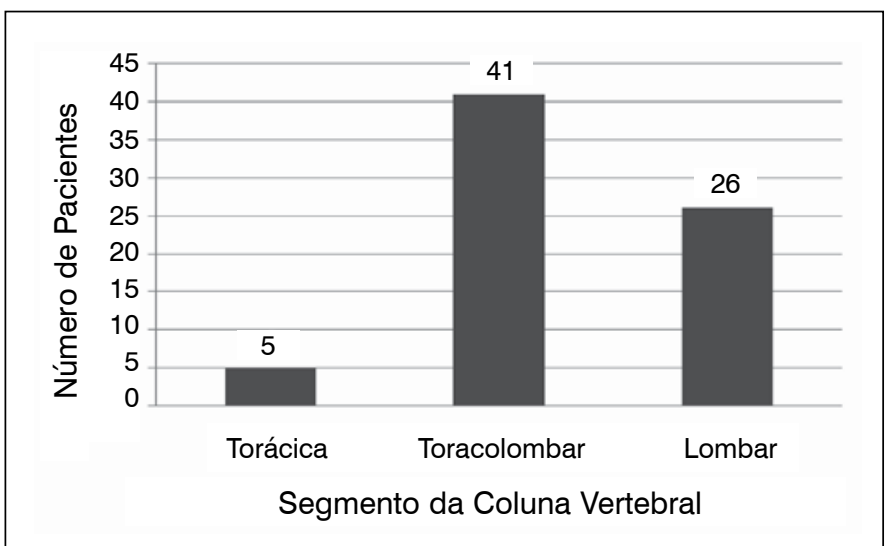

Figura 3. Distribuição das fraturas tipo explosão de acordo com o segmento biomecânico sagital.

Tabela 1. Comparação do estreitamento do canal vertebral (\%), nos pacientes com fraturas tipo explosão, nos segmentos torácico, transição toracolombar e lombar.

\begin{tabular}{c|c|c|c|c|c|c}
\hline $\begin{array}{c}\text { Segmento da } \\
\text { coluna vertebral }\end{array}$ & N & Média* $^{*}$ & $\begin{array}{c}\text { Desvio } \\
\text { padrão }\end{array}$ & Mín. & Mediana & Máx. \\
\hline Torácica $^{A}$ & 5 & 15,0 & 11,7 & 5 & 10,0 & 35 \\
\hline Toracolombar $^{B}$ & 41 & 29,5 & 17,3 & 5 & 25,0 & 72 \\
\hline Lombar $^{C}$ & 26 & 41,5 & 22,0 & 10 & 37,5 & 95 \\
\hline Total & 72 & 32,8 & 20,1 & 5 & 29 & 95
\end{tabular}

A. A porcentagem média de estreitamento do canal vertebral, causada pelo fragmento ósseo da fratura foi significante, testando segmento torácico e toracolombar $(p=0,046)$; B. Diferença significativa para comparação entre segmento torácico e lombar $(p=0,005)$; C. Diferença significativa entre segmento toracolombar e lombar $(p=0,026) ;{ }^{\star} p<0,05$; Teste Mann-Whitney.

Tabela 2. Comparação do colapso do corpo vertebral (\%), nos pacientes com fraturas tipo explosão, nos segmentos torácico, transição toracolombar e lombar.

\begin{tabular}{c|c|c|c|c|c|c}
\hline $\begin{array}{c}\text { Segmento da } \\
\text { coluna vertebral }\end{array}$ & N & Média* $^{*}$ & $\begin{array}{c}\text { Desvio } \\
\text { Padrão }\end{array}$ & Mín. & Mediana & Máx. \\
\hline Torácica $^{\mathrm{A}}$ & 5 & 34,3 & 24,3 & 0,4 & 37,8 & 66,7 \\
\hline Toracolombar $^{\mathrm{B}}$ & 41 & 35,5 & 14,5 & 14,3 & 32,3 & 82,4 \\
\hline Lombar $^{\mathrm{C}}$ & 26 & 32,4 & 15,4 & 4,5 & 37 & 60,0 \\
\hline Total & 72 & 34,3 & 15,4 & 0,4 & 31,4 & 82,4
\end{tabular}

A. A porcentagem média de colapso do corpo vertebral não apresentou significância no teste aplicado para o segmento torácico e toracolombar $(p=0,973)$; B. Sem diferença significativa para comparação entre segmento torácico e lombar $(p=0,735)$; $C$. Sem diferença significativa entre segmento toracolombar e lombar $(p=0,528) ;{ }^{*} p>0,05$; Teste de Mann-Whitney.
Tabela 3. Comparação entre o grau de cifose local, nos pacientes com fraturas tipo explosão, nos segmentos torácico, transição toracolombar e lombar.

\begin{tabular}{c|c|c|c|c|c|c}
\hline $\begin{array}{c}\text { Segmento da } \\
\text { coluna vertebral }\end{array}$ & N & Média* $^{*}$ & $\begin{array}{c}\text { Desvio } \\
\text { padrão }\end{array}$ & Mín. & Mediana & Máx. \\
\hline Torácico $^{A}$ & 5 & 28 & 11,6 & 10 & 31,0 & 40 \\
\hline Toracolombar $^{B}$ & 41 & 13,6 & 7,5 & 1 & 12,0 & 34 \\
\hline Lombar $^{C}$ & 26 & 0,23 & 13,7 & -30 & 3,5 & 22 \\
\hline Total & 72 & 9,8 & 13 & -30 & 10 & 40 \\
\hline
\end{tabular}

A. Houve significância no teste estatístico entre os segmentos torácico e toracolombar $(p=0,009)$; Diferença para comparação entre segmento torácico e lombar $(p<0,000)$; C. Diferença significativa entre segmento toracolombar e lombar $(p<0,000)$; ${ }^{*} p<0,05$; Teste Mann-Whitney

\section{DISCUSSÃO}

As fraturas da coluna torácica e lombar do tipo explosão são frequentes e estão geralmente associadas a traumas de grande energia cinética dissipada para a lesão. ${ }^{19}$ Muitos pacientes, que são vitimados por este tipo específico de fratura, são politraumatizados e apresentam alteração da função neurológica parcial ou completa, muitas vezes irreversível, independente do tratamento preconizado. ${ }^{1,18}$ Por isso, estudos que visam compreender a relação existente entre energia dissipada no trauma e os diversos fatores anatômicos e biomecânicos que influenciam no aparecimento e gravidade da fratura tipo explosão, permitem o raciocínio no que diz respeito ao tipo e momento do tratamento mais apropriado e prognóstico das lesões.

Os mecanismos de trauma mais comuns, descritos na literatura, são os de queda de altura e os acidentes automobilísticos. ${ }^{20-23}$ Por ter a característica de ser um segmento da coluna vertebral com maior mobilidade do que a coluna torácica e lombar baixa, a transição toracolombar tem sido a região com o maior incidência de fraturas, em todos os trabalhos analisados. ${ }^{22,24-27}$ Este achado também foi evidenciado no presente estudo.

O sexo masculino foi predominante no nosso trabalho, fato evidenciado também nas demais publicações. ${ }^{22,28,29}$

A determinação da gravidade das fraturas da coluna vertebral é fundamental e, por isso, tem sido o foco de estudos, na literatura, por vários autores. 1,18,30,31 Eles começaram a classificar as fraturas quanto a sua estabilidade. ${ }^{32-34} \mathrm{Com}$ a utilização da TC, constatou-se que o diagnóstico da fratura tipo explosão poderia ser feito com maior precisão, permitindo a avaliação objetiva da dimensão do canal vertebral comprometido por fragmentos ósseos. ${ }^{35,36}$ Baseadas nas alterações anatômicas e morfofuncionais, classificações das fraturas tipo explosão foram idealizadas para identificar os subtipos e facilitar a escolha do tratamento. ${ }^{1,18}$ Magerl et al. ${ }^{18}$ subdividiram as fraturas tipo explosão em três subgrupos (A3.1, A3.2 e A3.3), nos quais se considera a gravidade das lesões de forma crescente. No nosso estudo, as fratura da coluna vertebral do tipo explosão com maior incidência de casos foi a do subgrupo A3.1. Acreditamos que este achado seja decorrente de que a maioria dos casos de fraturas na coluna torácica e lombar tendem a ser de menor gravidade, independente do tipo ou subtipo de fratura.

Meves e Avanzi, ${ }^{30}$ fazendo uma avaliação estatística detalhada de 198 fraturas tipo explosão documentadas com tomografia computadorizada, encontrou média de estreitamento do canal de $42,1 \%$ e $62,4 \%$, para as regiões toracolombar e lombar, respectivamente, nos pacientes com déficit neurológico inicial. No presente estudo, a média do estreitamento do canal vertebral foi maior no segmento lombar $\left(L_{2}\right.$ a $\left.L_{5}\right)$, seguido da transição toracolombar $\left(T_{12}\right.$ e $\left.L_{1}\right)$ e torácico $\left(T_{1}\right.$ a $\left.T_{11}\right)$, respectivamente. Acreditamos que este fato tenha ocorrido devido às diferenças anatômicas e biomecânicas que existem entre os diferentes segmentos da coluna. O eixo biomecânico sagital passa anteriormente ao segmento torácico e transição toracolombar. No segmento lombar, o mesmo eixo passa sob a porção média e posterior dos corpos vertebrais lombares, que encontram-se em área de lordose. ${ }^{14}$ Isto, aliado à anatomia da região, permitem a compreensão dos motivos pelos quais a maior média de estreitamento ocorre no segmento lombar. 
Devemos recordar, por outro lado, que a presença da cauda equina é considerada um atenuante na gravidade das lesões que ocorrem na região lombar baixa. Nos segmentos torácicos e de transição toracolombar, a presença da medula espinal e cone medular, respectivamente, podem ser causadores de lesões neurológicas mais graves. No presente estudo, as fraturas tipo explosão não apresentaram média de estreitamento do canal vertebral elevadas, nestes referidos segmentos, o que atenua uma maior quantidade de fraturas tipo explosão associadas à déficit neurológico.

A cifose e o colapso decorrentes de fraturas da coluna torácica e lombar tipo explosão, têm sido considerados critérios importantes para considerar a escolha da conduta terapêutica., ${ }^{1,37-43}$ No presente estudo, foi observado que a média da cifose, decorrente das fraturas tipo explosão, nos diferentes segmentos biomecânicos sagitais avaliados, foi estatisticamente significante. Porém, o colapso do corpo vertebral não apresentou diferença estatisticamente significativa, quando os valores entre os diferentes segmentos biomecânicos sagitais foram analisados. Verifica-se, com base nos achados do presente estudo, que as curvas anatômicas (cifose torácica e lordose lombar), possuem influência direta no grau de cifose da região fraturada, quando o trauma axial é dissipado sob o eixo biomecânico da coluna. Acreditamos que a comparação do colapso vertebral da vértebra fraturada, entre os grupos avaliados, não tenha sido estatisticamente significativa, em decorrência do fato de que a maioria das fraturas foram classificadas como A3.1, ou seja, fragmentação apenas da porção superior do corpo da vértebra.

\section{REFERÊNCIAS}

1. Denis F. The three column spine and its significance in the classification of acute thoracolumbar spinal injuries. Spine (Phila Pa 1976). 1983;8(8):817-31.

2. Holdsworth FW. Fractures, dislocations and fractures-dislocations of the spine. J. Bone Joint Surg. 1963;45B(1):6-20.

3. Trafton PG, Boyd CA Jr. Computed tomography of thoracic and lumbar spine injuries. J Trauma. 1984;24(6):506-15.

4. Herbert $S$, Xavier R, Pardini Junior AG, Barros Filho TE. Ortopedia e traumatologia, princípios e prática. 3a ed. São Paulo: Artmed; 2003.

5. Avanzi O, Salomão JCA, Dezen E, Pinto Filho WC. Fratura da coluna em crianças: estudo de 38 casos. Rev Bras Ortop. 1993:28(3):105-11.

6. Puertas EB, Chagas JC, Mercúrio R, Milani A. Fraturas da coluna vertebral na região toracolombar: estudo de 36 pacientes. Rev Bras Ortop. 1991;26(4):196-200.

7. Solino JL, Melo MF, Silva DH, Elias N. Traumatismo da coluna vertebral: avaliação de etiologia, incidência e freqüência. Rev Bras Ortop. 1990;25(6):185-90.

8. Zardo E, Surmay MJ. Lesões da coluna vertebral. In: Herbert S, Xavier R, editores. Ortopedia e traumatologia - Princípios e prática. Porto Alegre: Artes Médicas; 1995. p. 384-401.

9. Oner FC, Wood KB, Smith JS, Shaffrey $\mathrm{Cl}$. Therapeutic decision making in thoracolumbar spine trauma. Spine (Phila Pa 1976). 2010;35(Suppl 21):S235-44

10. Alpantaki K, Bano A, Pasku D, Mavrogenis AF, Papagelopoulos PJ, Sapkas GS, et al. Thoracolumbar burst fractures: a systematic review of management. Orthopedics. 2010;33(6):422-9.

11. Rezende R, Avanzi O. The importance of injury severity score (ISS) in the management of thoracolumbar burst fracture. Rev Col Bras Cir. 2009;36(1):9-13

12. Seybold EA, Sweeney CA, Fredrickson BE, Warhold LG, Bernini PM. Functional outcome of low lumbar burst fractures. A multicenter review of operative and nonoperative treatment of L3-L5. Spine (Phila Pa 1976). 1999;24(20):2154-61.

13. White AA III, Panjabi MM. Clinical Biomechanics of the Spine. 2nd ed. Philadelphia: Lippincott; 1990.

14. Tisot RA, Avanzi O. Fratura da coluna vertebral tipo explosão na área da cauda equina: correlação entre função neurológica e alterações estruturais no canal vertebral. Acta Ortop Bras. 2008;16(2):85-8

15. Benhardt M, White AA 3rd, Panjabi MM. Biomechanichal considerations of spinal stability. In: Herkowitz HN, Garfin SR, Eismont FJ, Bell GR, Balderston RA, editors. Rothman Simeone. The spine. 5th ed. Philadelphia, Pensilvania: Elsevier; 2006. p. 132-56.

16. Cobb JR. Outline for the study of scoliosis. Instr Course Lect. 1948;5:261-75.

17. Willén J, Lindahl S, Nordwall A. Unstable thoracolumbar fractures. A comparative clinical study of conservative treatment and Harrington instrumentation. Spine (Phila Pa 1976). 1985; 10(2):111-22.

18. Magerl F, Aebi M, Gertzbein SD, Harms J, Nazarian S. A comprehensive classification of thoracic and lumbar injuries. Eur Spine J. 1994;3(4):184-201.

19. Meves R, Avanzi O. Correlation among canal compromise, neurologic deficit, and injury severity in thoracolumbar burst fractures. Spine (Phila Pa 1976). 2006:31(18):2137-41.

20. Oliveira PAS, Pires JV, Borges Filho JMM. Traumatismo da coluna torácica e lombar: Avaliação epidemiológica. Rev Bras Ortop. 1996;31(9):771-6.

21. Saal JS. General principles of diagnostic testing as related to painful lumbar spine disorders: a critical appraisal of current diagnostic techniques. Spine (Phila Pa 1976). 2002;27(22):2538-45

22. Mumford J, Weinstein JN, Spratt KF, Goel VK. Thoracolumbar burst fractures. The
Embora não tenha havido diferença estatisticamente significativa, o segmento lombar apresentou a menor média de colapso. Este achado pode ter sido decorrente da aferição de colapso ter sido realizada apenas na parede anterior da vértebra fraturada. Como há uma curva anatômica lordótica no segmento lombar, o trauma axial, quando dissipado nesta região, biomecanicamente acomete mais a porção média e posterior das vértebras lombares.

Acreditamos que a elaboração de mais estudos que possam comparar o tratamento das fraturas da coluna torácica e lombar tipo explosão, em diferentes segmentos biomecânicos e anatômicos, sejam valiosos e colaborarão para que as indicações de tratamento deste tipo de fratura sejam mais criteriosas e coesas.

\section{CONCLUSÃO}

As fraturas da coluna torácica e lombar tipo explosão, embora apresentem características semelhantes, independente do local em que ocorrem, sofrem influência direta do segmento biomecânico sagital, no que diz respeito às alterações estruturais que ocorrem na vértebra fraturada. O estreitamento do canal vertebral, causado pelo fragmento da fratura, foi significativamente maior no segmento lombar. Já a cifose na região fraturada, foi significativamente maior no segmento torácico.

Todos os autores declaram não haver nenhum potencial conflito de interesses referente a este artigo.

clinical efficacy and outcome of nonoperative management. Spine (Phila Pa 1976) 1993;18(8):955-70.

23. Cantor JB, Lebwohl NH, Garvey T, Eismont FJ. Nonoperative management of stable thoracolumbar burst fractures with early ambulation and bracing. Spine (Phila Pa 1976). 1993;18(8):971-6.

24. Krompinger WJ, Fredrickson BE, Mino DE, Yuan HA. Conservative treatment of fractures of the thoracic and lumbar spine. Orthop Clin North Am. 1986;17(1):161-70.

25. Wood K, Buttermann G, Mehbod A, GarveyT, Jhanjee R, Sechriest V. Operative compared with nonoperative treatment of a thoracolumbar burst fracture without neurological deficit. A prospective, randomized study. J Bone Joint Surg Am. 2003;85(5):773-81.

26. Shen WJ, Shen YS. Nonsurgical treatment of three-column thoracolumbar junction burst fractures without neurologic deficit. Spine (Phila Pa 1976). 1999;24(4):412-5.

27. Esperidião AP, Umeta RGS, Caffaro MFS, Meves R, Landim E, Avanzi O. Avaliação radiográfica do colapso sagital na fratura toracolombar tipo B de Magerl. Coluna/Columna. 2010;9(4):387-93.

28. Cunha FM, Menezes CM, Guimarães EP. Lesões traumáticas da coluna torácica e lombar. Rev Bras Ortop. 2000;35(1):17-22.

29. Jorge FF, Baldini APS, Barros Filho TP. Trauma raquimedular: aspectos epidemiológicos, impacto social e prevenção. Acta Ortop Bras. 1995;3(1):1-4.

30. Meves R, Avanzi O. Correlation between neurological deficit and spinal canal compromise in 198 patients with thoracolumbar and lumbar fractures. Spine (Phila Pa 1976). 2005;30(7):787-91.

31. Tisot RA, Avanzi O. Laminar fractures as a severity marker in burst fractures of the thoracolumbar spine. J Orthop Surg (Hong Kong). 2009;17(3):261-4.

32. Nicoll EA. Fractures of the dorso-lumbar spine. J Bone Joint Surg Br. 1949;31(3):376-94.

33. Holdsworth FW, Hardy A. Early treatment of paraplegia from fractures of the thoracolumbar spine. J Bone Joint Surg Br. 1953;35-B(4):540-50.

34. Whitesides TE Jr. Traumatic kyphosis of the thoracolumbar spine. Clin Orthop Relat Res. 1977;(128):78-92.

35. Colley DP, Dunsker SB. Traumatic narrowing of the dorsolumbar spinal canal demonstrated by computed tomography. Radiology. 1978;129(1):95-8.

36. Avanzi $\mathrm{O}$, Meves R. Avaliação tomográfica do fragmento retropulsão nas fraturas toracolombares tipo explosão. In: $11^{\circ}$ Congresso de Ortopedia e Traumatologia do Estado de São Paulo (COTESP), Atibaia, SP, 2005.

37. Defino HLA, Fuentes ARR, Remondi PH, Vallim EC. Tratamento conservador da coluna toracolombar. Rev Bras Ortop. 2000;35(8):301-8.

38. CotrelY, Dubousset J, Guillaumat M. New universal instrumentation in spinal surgery. Clin Orthop Relat Res. 1988;227:10-23.

39. Bohlman HH. Treatment of fractures and dislocations of the thoracic and lumbar spine. J Bone Joint Surg Am. 1985;67(1):165-9.

40. Bradford DS, Akbarnia BA, Winter RD, Seljeskog EC. Surgical stabilization of fractures and fracture-dislocations of the thoracic spine. Spine (Phila Pa 1976). 1977;2(2):185-96.

41. Levine AM, Edwards CC. Low lumbar burst fractures. Reduction and stabilization using the modular spine fixation system. Orthopedics. 1988;11(10):1427-32.

42. Akbarnia BA, Crandall DG, Burkus K, Matthews T. Use of long rods and a short arthrodesis for burst fractures of the thoracolumbar spine. A long term follow-up study. J Bone Joint Surg Am. 1994:76(11):1629-35.

43. Akalm S, Kiş $M$, Benli IT, Citak M, Mumcu EF, Tüzüner $M$. Results of the $A O$ spinal internal fixator in the surgical treatment of thoracolumbar burst fractures. Eur Spine J. 1994;3(2):102-6. 